\title{
INVERSE PROBLEMS FOR INVARIANT ALGEBRAIC CURVES: EXPLICIT COMPUTATIONS
}

\author{
COLIN CHRISTOPHER ${ }^{1}$, JAUME LLIBRE $^{2}$, CHARA PANTAZI $^{3}$ AND \\ SEBASTIAN WALCHER ${ }^{4}$
}

\begin{abstract}
Given an algebraic curve in the complex affine plane, we describe how to determine all planar polynomial vector fields which leave this curve invariant. If all (finite) singular points of the curve are nondegenerate, we give an explicit expression for these vector fields. In the general setting we provide an algorithmic approach, and as an alternative we discuss sigma processes.
\end{abstract}

\section{INTRODUCTION}

The question to determine the invariant algebraic curves of a given planar polynomial vector field, or to decide that no such curves exist, is part of a problem set forth by Poincaré, and is also essential in deciding whether the vector field admits a Darboux integrating factor. While there are many partial answers for this problem, a complete solution still seems beyond reach.

A solution of the inverse problem - to determine all polynomial vector fields that admit a prescribed collection of invariant algebraic curves (or just one possibly reducible curve) - seems to be essential in order to obtain a proper understanding of the situation. In this paper we present a solution to this inverse problem, using mainly results and tools from elementary commutative algebra. Our results indicate that it is sensible to consider the affine plane in its own right, as well as a subset of the projective plane. For the sake of completeness and to emphasize that our approach works very naturally, the paper also includes streamlined alternative proofs for some theorems that have been published previously.

1991 Mathematics Subject Classification. Primary 34C05, 34A34, 34C14.

Key words and phrases. polynomial differential system, invariant algebraic curve.

J.L. and Ch.P. are partially supported by a MEC/FEDER grant MTM 2005-06098C02-01 and by a CIRIT grant number 2001SGR 00550. Ch.P. is additionally partially supported by a MEC/FEDER grant MTM2006-00478. C.C.and S.W. acknowledge the hospitality and support of the CRM and Mathematics Department at Universitat Autònoma de Barcelona during visits when this manuscript was prepared. 
The vector fields admitting the given curve obviously form a linear space. We identify two subspaces that contain only vector fields of a known explicit form and we show that the quotient by either of these subspaces is finite dimensional. Assuming certain nondegeneracy conditions for the affine curve, we show that the whole linear space equals one of these subspaces. Generally we give dimension estimates, and we describe an algorithmic approach to determining the quotient space (indicating by examples that this is feasible). Finally we discuss blow-ups. In principle these allow to transform every affine curve to one that satisfies the nondegeneracy conditions. This opens another path towards the determination of the quotient space, and provides more structural insight.

\section{BASICS AND KNOWN FACTS}

We consider a complex polynomial vector field

$$
X=P \frac{\partial}{\partial x}+Q \frac{\partial}{\partial y},
$$

sometimes also written as

$$
X=\left(\begin{array}{c}
P \\
Q
\end{array}\right)
$$

and a non-constant polynomial $f$ with prime factors $f_{1}, \ldots, f_{r}$.

The complex zero set of $f$ (which is generally a reducible curve in $\mathbb{C}^{2}$ ) is invariant for the vector field if and only if there is a polynomial $L$ (called the cofactor of $f$ ) such that

$$
X f=L \cdot f, \quad \text { or } P \cdot f_{x}+Q \cdot f_{y}=L \cdot f
$$

equivalently there are polynomials $L_{1}, \ldots, L_{r}$ such that

$$
X f_{i}=L_{i} \cdot f_{i}, \quad 1 \leq i \leq r .
$$

We will briefly say that in this case the vector field $X$ admits $f$, or admits the curve given by $f=0$. In the following we will assume $f=f_{1} \cdots f_{r}$, which causes no loss of generality (therefore $L=L_{1}+\cdot+L_{r}$ ). The respective zero sets of $f$ and the $f_{i}$ in $\mathbb{C}^{2}$ will be denoted by $C$ and $C_{i}$. As usual we call a point $z$ with $f(z)=f_{x}(z)=f_{y}(z)=0$ a singular point of $C$, and similarly for the $C_{i}$. The Hamiltonian vector field of $f$ is defined by

$$
X_{f}=-f_{y} \frac{\partial}{\partial x}+f_{x} \frac{\partial}{\partial y} .
$$

For a given $f$ the vector fields admitting $f$ form a linear space $\mathcal{V}_{f}$. Our goal is to determine this space precisely. First, we collect some facts and 
properties that are known from previous work. (As for references, see [2], [3], [8], [10].) Clearly vector fields of the form

$$
X=a \cdot X_{f}+f \cdot \tilde{X}
$$

with an arbitrary polynomial $a$ and an arbitrary polynomial vector field $\widetilde{X}$ admit the invariant curve $C$. These vector fields will be called the trivial vector fields admitting $f$. They form a subspace of $\mathcal{V}_{f}$ which will be called $\mathcal{V}_{f}^{0}$. For reducible $f$ one can refine this to see that all vector fields of the form

$$
X=\sum_{i} a_{i} \frac{f}{f_{i}} \cdot X_{f_{i}}+f \cdot \widetilde{X}
$$

admit $f$. These vector fields form a subspace of $\mathcal{V}_{f}$ which will be denoted $\mathcal{V}_{f}^{1}$. Note that $\mathcal{V}_{f}^{0} \subseteq \mathcal{V}_{f}^{1}$. In "generic" geometric settings one has $\mathcal{V}_{f}=\mathcal{V}_{f}^{1}$; see [3] and an improvement in [2, Theorem 3.4] (in conjunction with [2, Theorem 3.6]). We will reprove the latter result using a different approach.

\section{The IDEAL OF COFACTORS}

In this section we clarify the structure of the linear space $\mathcal{V}_{f}$ of polynomial vector fields admitting $f$. In part the exposition is parallel to [10], Section 2 , with some improvements and streamlining. We start with an auxiliary result.

Lemma 1. Let $g$ be a nonconstant polynomial with no multiple prime factors, and $s$ be a greatest common divisor of the partial derivatives $g_{x}$ and $g_{y}$. If $s$ is not constant then $g$ is constant and nonzero on every irreducible component of the vanishing set of $s$. In particular, $g$ and $s$ have no common zeros and there exist polynomials $u$ and $v$ such that $u \cdot s+v \cdot g=1$.

Proof. Let $C^{*}$ be an irreducible component of the vanishing set of $s$, corresponding to a prime factor $s^{*}$ of $s$, and $\gamma=\left(\gamma_{1}, \gamma_{2}\right)$ a local parametrization of $C^{*}$ near a nonsingular point. Since the partial derivatives of $g$ vanish on $C^{*}$, one has

$$
\frac{d}{d t}(g(\gamma(t)))=g_{x}(\gamma(t)) \dot{\gamma}_{1}(t)+g_{y}(\gamma(t)) \dot{\gamma}_{2}(t)=0
$$

and thus $g$ is locally constant on $C^{*}$, which implies constancy since $C^{*}$ is irreducible.

Now assume that $g=0$ on $C^{*}$. Then the vanishing set of $s^{*}$ is contained in the vanishing set of $g$, and by irreducibility and Hilbert's Nullstellensatz one finds that $s^{*}$ divides $g$. Thus $g=s^{*} \cdot h$ with $s^{*}$ and $h$ relatively prime. 
This implies

$$
\begin{aligned}
& g_{x}=s^{*} \cdot h_{x}+s_{x}^{*} \cdot h \\
& g_{y}=s^{*} \cdot h_{y}+s_{y}^{*} \cdot h .
\end{aligned}
$$

Therefore $s^{*}$ divides both its partial derivatives, which is impossible from degree considerations; we have a contradiction.

The second assertion is clear with Hilbert's Nullstellensatz.

Now we turn to equation (2) and consider the cofactor $L$. For given $f$ the cofactors of all vector fields admitting $f$ clearly form an ideal. By definition of ideal quotients one obtains

$$
L \in\left\langle f_{x}, f_{y}\right\rangle:\langle f\rangle
$$

as a necessary and sufficient condition for the existence of $P$ and $Q$ such that (2) holds. We first clarify the role of the trivial vector fields admitting $f$.

Proposition 2. A polynomial vector field $X$ satisfies equation (2) with $L \in\left\langle f_{x}, f_{y}\right\rangle$ if and only if $X \in \mathcal{V}_{f}^{0}$.

Proof. Evaluating (2) for $L=\widetilde{P} \cdot f_{x}+\widetilde{Q} \cdot f_{y}$ one finds

$$
(P-f \cdot \widetilde{P}) \cdot f_{x}+(Q-f \cdot \widetilde{Q}) \cdot f_{y}=0,
$$

hence

$$
\begin{aligned}
P-f \cdot \widetilde{P} & =\tilde{a} \cdot\left(-f_{y} / s\right) \\
Q-f \cdot \widetilde{Q} & =\tilde{a} \cdot\left(f_{x} / s\right)
\end{aligned}
$$

with a g.c.d $s$ of the partial derivatives of $f$, and some polynomial $\tilde{a}$. Thus we have a representation

$$
X=\frac{\tilde{a}}{s} \cdot X_{f}+f \cdot \tilde{X}
$$

and with $\tilde{a}=u \cdot s \cdot \tilde{a}+v \cdot f \cdot \tilde{a}$ from Lemma 1 we obtain

$$
X=u \tilde{a} \cdot X_{f}+f \cdot\left(v \tilde{a} \cdot \frac{1}{s} X_{f}+\tilde{X}\right)
$$

and the proof is finished.

By standard arguments we obtain the first Corollary.

Corollary 3. The map sending a vector field admitting $f$ to its cofactor induces an isomorphism

$$
\mathcal{V}_{f} / \mathcal{V}_{f}^{0} \cong\left(\left\langle f_{x}, f_{y}\right\rangle:\langle f\rangle\right) /\left\langle f_{x}, f_{y}\right\rangle .
$$


Corollary 4. For $r>1$ let $p_{i}:=X_{f_{i}}\left(f / f_{i}\right)=-\left\{f_{i}, f / f_{i}\right\}$, where $\{.,$. denotes the standard Poisson bracket. Then $X \in \mathcal{V}_{f}^{1}$ if and only if

$$
L \in<f_{x}, f_{y}, p_{1}, \cdots, p_{r-1}>
$$

holds for the associated cofactor, and

$$
\mathcal{V}_{f} / \mathcal{V}_{f}^{1} \cong\left(<f_{x}, f_{y}>:<f>\right) /<f_{x}, f_{y}, p_{1}, \cdots, p_{r-1}>.
$$

Proof. Let $Y_{i}:=f / f_{i} \cdot X_{f_{i}}$, with associated cofactor $L_{i}=\frac{1}{f_{i}} X_{f_{i}}(f)=$ $X_{f_{i}}\left(f / f_{i}\right)=p_{i}$. Thus $X=\sum a_{i} Y_{i}+f \tilde{X} \in \mathcal{V}_{f}^{1}$ implies $L \in<f_{x}, f_{y}, p_{1}, \cdots, p_{r}>$, and $p_{r}$ can be discarded in view of $p_{1}+\cdots+p_{r}=0$.

For the reverse inclusion assume that $X$ has cofactor $L=\sum b_{i} p_{i}+u \cdot f_{x}+$ $v \cdot f_{y}$, and set $\hat{X}=\sum b_{i} Y_{i}+f \cdot\left(\begin{array}{c}u \\ v\end{array}\right)$. Then $C$ is invariant for the vector field $X-\hat{X}$ with zero cofactor, thus $X-\hat{X}=\frac{\tilde{a}}{s} X_{f}$, as in the proof of Proposition 2. Then the arguments in this proof show $X-\tilde{X} \in \mathcal{V}_{f}^{0} \subset \mathcal{V}_{f}^{1}$.

In particular Proposition 2 shows that $\mathcal{V}_{f}=\mathcal{V}_{f}^{0}$ when $\left\langle f_{x}, f_{y}\right\rangle=\langle 1\rangle$. In the following we will proceed as in [10], assuming that $\left\langle f_{x}, f_{y}\right\rangle \neq\langle 1\rangle$ and considering a primary decomposition

$$
\left\langle f_{x}, f_{y}\right\rangle=\mathbf{q}_{1} \cap \cdots \cap \mathbf{q}_{s}
$$

with primary ideals $\mathbf{q}_{i}$ and associated prime ideals $\mathbf{p}_{i}$. It is well known that either $\mathbf{p}_{i}$ is generated by an irreducible polynomial (and thus corresponds to an irreducible curve), or that $\mathbf{p}_{i}$ is maximal and corresponds to a point in the complex plane. By a standard result from commutative algebra

$$
\left\langle f_{x}, f_{y}\right\rangle:\langle f\rangle=\left(\mathbf{q}_{1}:\langle f\rangle\right) \cap \cdots \cap\left(\mathbf{q}_{s}:\langle f\rangle\right)
$$

see [10]. We quote one more result from [10].

Proposition 5. Let the notation be as in (5). Then $\mathbf{q}_{i}:\langle f\rangle \neq \mathbf{q}_{i}$ if and only if $\mathbf{p}_{i}$ corresponds to a singular point of $C$. In particular $\mathcal{V}_{f}=\mathcal{V}_{f}^{0}$ if $C$ has no singular point.

Now assume that $C$ has singular points, and that precisely the ideals $\mathbf{q}_{1}, \ldots, \mathbf{q}_{m}$, with some $m \geq 1$, correspond to these singular points. Let

$$
\mathbf{a}:=\mathbf{q}_{1} \cap \ldots \cap \mathbf{q}_{m}, \quad \mathbf{b}:=\mathbf{q}_{m+1} \cap \ldots \cap \mathbf{q}_{s}
$$

with $\mathbf{b}:\langle f\rangle=\mathbf{b}$, and understanding $\mathbf{b}=\mathbb{C}[x, y]$ in case $s=m$. Lemma 1 shows that $\mathbf{a}$ and $\mathbf{b}$ have no common zero, thus $\mathbf{a}+\mathbf{b}=\mathbb{C}[x, y]$. The same argument shows

$$
\mathbf{q}_{i}+\bigcap_{j \neq i} \mathbf{q}_{j}=\mathbb{C}[x, y], \quad 1 \leq i \leq m .
$$


It should be noted that $\mathbb{C}[x, y] / \mathbf{a}$ is finite dimensional, see [10]. The following result is an improved version of Theorem 2.3 of [10] which clarifies the structure of $\mathcal{V}_{f}$ and provides a basis for computations.

Theorem 6. Let the hypotheses and notation be as above.

(a) The maps

$$
\begin{aligned}
& \left(\left\langle f_{x}, f_{y}\right\rangle:\langle f\rangle\right) /\left\langle f_{x}, f_{y}\right\rangle \rightarrow(\mathbf{a}:\langle f\rangle) / \mathbf{a} \\
& g+\left\langle f_{x}, f_{y}\right\rangle \mapsto g+\mathbf{a} \\
& \text { and } \\
& \left(\left\langle f_{x}, f_{y}\right\rangle:\langle f\rangle\right) /\left\langle f_{x}, f_{y}\right\rangle \rightarrow \bigoplus_{1 \leq i \leq m}\left(\mathbf{q}_{i}:\langle f\rangle\right) / \mathbf{q}_{i} \\
& g+\left\langle f_{x}, f_{y}\right\rangle \quad \mapsto \quad\left(g+\mathbf{q}_{1}, \ldots, g+\mathbf{q}_{m}\right)
\end{aligned}
$$

are isomorphisms.

(b) The kernel of the map

$$
\begin{aligned}
\mathbb{C}[x, y] / \mathbf{a} & \rightarrow \mathbb{C}[x, y] / \mathbf{a} \\
g+\mathbf{a} & \mapsto f \cdot g+\mathbf{a}
\end{aligned}
$$

is equal to $(\mathbf{a}:\langle f\rangle) / \mathbf{a}$. This gives rise to an isomorphism

$$
\left(\left\langle f_{x}, f_{y}\right\rangle:\langle f\rangle\right) /\left\langle f_{x}, f_{y}\right\rangle \cong \mathbb{C}[x, y] /\left\langle f, f_{x}, f_{y}\right\rangle .
$$

(c) Assume that $f_{x}=s \cdot h_{1}, f_{y}=s \cdot h_{2}$ with a g.c.d. $s$ of the partial derivatives, and $\mathbf{c}:=\left\langle h_{1}, h_{2}\right\rangle$. Then $\mathbb{C}[x, y] / \mathbf{c}$ has finite dimension, and the invariance condition (2) holds if and only if

$$
L=s \cdot \widehat{L} \quad \text { with } \quad \widehat{L} \in \mathbf{c}:\langle f\rangle .
$$

The map

$$
\begin{aligned}
\mathbb{C}[x, y] / \mathbf{c} & \rightarrow \mathbb{C}[x, y] / \mathbf{c} \\
g+\mathbf{c} & \mapsto f \cdot g+\mathbf{c}
\end{aligned}
$$

has kernel $(\mathbf{c}:\langle f\rangle) / \mathbf{c}$.

Proof. The Chinese Remainder Theorem implies that

$$
\begin{aligned}
\mathbb{C}[x, y] /(\mathbf{a} \cap \mathbf{b}) & \rightarrow \mathbb{C}[x, y] / \mathbf{a} \oplus \mathbb{C}[x, y] / \mathbf{b} \\
g+\mathbf{a} \cap \mathbf{b} & \mapsto(g+\mathbf{a}, g+\mathbf{b})
\end{aligned}
$$

is an isomorphism. For an ideal $\mathbf{h} \supseteq \mathbf{a} \cap \mathbf{b}$ this induces the corresponding isomorphism

$$
\mathbf{h} /(\mathbf{a} \cap \mathbf{b}) \cong(\mathbf{h}+\mathbf{a}) / \mathbf{a} \oplus(\mathbf{h}+\mathbf{b}) / \mathbf{b} .
$$


Now $\left\langle f_{x}, f_{y}\right\rangle:\langle f\rangle=(\mathbf{a}:\langle f\rangle) \cap \mathbf{b}$, and therefore (recalling $\left.\mathbf{a}+\mathbf{b}=\mathbb{C}[\mathbf{x}, \mathbf{y}]\right)$

$$
\begin{aligned}
& \left(\left\langle f_{x}, f_{y}\right\rangle:\langle f\rangle\right)+\mathbf{a}=\mathbf{a}:\langle f\rangle \\
& \left(\left\langle f_{x}, f_{y}\right\rangle:\langle f\rangle\right)+\mathbf{b}=\mathbf{b} .
\end{aligned}
$$

Using (7) with $\mathbf{h}=\left\langle f_{x}, f_{y}\right\rangle:\langle f\rangle$ shows the first assertion of $(a)$. The second assertion of $(a)$ follows readily with

$$
\mathbb{C}[x, y] /\left(\mathbf{q}_{1} \cap \ldots \cap \mathbf{q}_{m}\right) \cong \bigoplus_{1 \leq i \leq m} \mathbb{C}[x, y] / \mathbf{q}_{i}
$$

and analogous arguments.

The kernel of the map in part (b) equals $(\mathbf{a}+\langle f\rangle) / \mathbf{a}$ by definition. To prove the isomorphism property, note that this map between finite dimensional vector spaces has image $(\langle f\rangle+\mathbf{a}) / \mathbf{a}$. Consideration of the cokernel yields

$$
\begin{aligned}
(\mathbf{a}:\langle f\rangle) / \mathbf{a} & \cong(\mathbb{C}[x, y] / \mathbf{a}) /((\langle f\rangle+\mathbf{a}) / \mathbf{a}) \\
& \cong \mathbb{C}[x, y] /(\langle f\rangle+\mathbf{a}) .
\end{aligned}
$$

In view of (a) there remains to show that

$$
\left\langle f, f_{x}, f_{y}\right\rangle=\langle f\rangle+\mathbf{a}=\langle f\rangle+\mathbf{a} \cap \mathbf{b} .
$$

But by Lemma $1, f$ and $\mathbf{b}$ have no common zeros, hence there are polynomials $g^{*} \in \mathbb{C}[x, y]$ and $b^{*} \in \mathbf{b}$ such that $g^{*} \cdot f+b^{*}=1$. Now for $g \cdot f+a \in\langle f\rangle+\mathbf{a}$ one finds

$$
\begin{aligned}
g \cdot f+a & =(g \cdot f+a)\left(g^{*} \cdot f+b^{*}\right) \\
& =\left(g \cdot g^{*} \cdot f+a \cdot g^{*}+b^{*} \cdot g\right) \cdot f+a \cdot b^{*} \in\langle f\rangle+\mathbf{a} \cap \mathbf{b} .
\end{aligned}
$$

So statement (b) follows.

Finally for statement (c) Lemma 1 shows the assertion about the invariance condition. The rest is then clear.

Corollary 7. For any polynomial $f$ with simple prime factors, the dimension of $\mathcal{V}_{f} / \mathcal{V}_{f}^{0}$ is finite and equal to the dimension of any of the spaces in Theorem 6 .

Proof. This follows from Corollary 3 and Theorem 6 if $\left\langle f_{x}, f_{y}\right\rangle \neq\langle 1\rangle$ and directly in the remaining case.

Some general consequences of Proposition 5 and Corollary 7 can be stated in explicit form. Recall that a singular point of $C$ has multiplicity one if and only if its associated ideal in the primary decomposition (5) is maximal; other characterizations are given by invertibility of the Hessian of $f$, or by a local transversality condition for intersections, see [10].

Theorem 8. The following statements hold. 
(a) The dimension of $\mathcal{V}_{f} / \mathcal{V}_{f}^{0}$ is greater than or equal to the number of singular points of $C$, with equality holding if every singular point of $C$ has multiplicity one. In particular $\mathcal{V}_{f}=\mathcal{V}_{f}^{0}$ when $C$ has no singular points.

(b) If $C$ has the singular points $z_{1}, \ldots, z_{m}$, all of multiplicity one, then the map

$$
\begin{aligned}
\mathcal{V}_{f} / \mathcal{V}_{f}^{0} & \rightarrow \mathbb{C}^{m} \\
X+\mathcal{V}_{f}^{0} & \mapsto\left(L\left(z_{1}\right), \ldots, L\left(z_{m}\right)\right)
\end{aligned}
$$

(with $L$ the cofactor of $f$ for the vector field $X$ ) is a well-defined isomorphism of vector spaces.

(c) If all $C_{i}$ are smooth, and if all intersections of distinct irreducible curves have multiplicity one, then $\mathcal{V}_{f}=\mathcal{V}_{f}^{1}$.

Proof. Statement (a) is obvious.

For statement (b), first note that the cofactor of a trivial vector field vanishes at any singular point of $C$ by Proposition 2, hence the map is welldefined. Now fix a singular point $z_{i}$. Since $z_{i}$ is simple, the corresponding ideal $\mathbf{q}_{i}$ is maximal, and necessarily $\mathbf{q}_{i}:\langle f\rangle=\mathbb{C}[x, y]$. Therefore there exists a vector field $Y_{i}$ admitting $f$ with cofactor $K_{i}$ and $K_{i}\left(z_{i}\right) \neq 0$. Multiplying $Y_{i}$ by suitable polynomials yields a vector field whose cofactor does not vanish at $z_{i}$ but vanishes at every other $z_{j}$. This finishes the proof of statement (b).

For statement (c) there only remains to show for that every singular point $z$ there exists an $X_{z} \in \mathcal{V}_{f}^{1}$ whose cofactor $L_{z}$ does not vanish in $z$. Now $z$ is a common zero of two distinct polynomials $f_{i}$ and $f_{j}$, and $f_{k}(z) \neq 0$ for all other $k$. Define

$$
X_{z}:=\frac{f}{f_{i}} X_{f_{i}}, \quad \text { with } \quad L_{z}=\sum_{\ell \neq i} \frac{f}{f_{i} f_{\ell}} X_{f_{i}}\left(f_{\ell}\right),
$$

then $L_{z}(z)$ is a nonzero multiple of $X_{f_{i}}\left(f_{j}\right)(z)$, and the latter is nonzero because of the transversality condition.

Statement (c) of this theorem shows that $\mathcal{V}_{f}=\mathcal{V}_{f}^{1}$ if certain geometric nondegeneracy conditions hold for the curves. This was also proved in [2], using a different approach.

For the dimension of $\mathcal{V}_{f} / \mathcal{V}_{f}^{1}$ we obtain an explicit expression.

Proposition 9. If $\mathbb{C}[x, y] /\left\langle f_{x}, f_{y}\right\rangle$ is finite dimensional then $\operatorname{dim} \mathcal{V}_{f} / \mathcal{V}_{f}^{1}=\operatorname{dim} \mathbb{C}[x, y] /\left\langle f_{x}, f_{y}, p_{1}, \cdots, p_{r-1}\right\rangle-\operatorname{dim}\left\langle f, f_{x}, f_{y}\right\rangle /\left\langle f_{x}, f_{y}\right\rangle$.

Proof. ¿From Corollary 4 we know that

$$
\mathcal{V}_{f} / \mathcal{V}_{f}^{1} \cong\left(\left\langle f_{x}, f_{y}\right\rangle:\langle f\rangle\right) /\left\langle f_{x}, f_{y}, p_{1}, \cdots, p_{r-1}\right\rangle \text {. }
$$


Now consider the linear map

$$
\mathbb{C}[x, y] /\left\langle f_{x}, f_{y}, p_{1}, \cdots, p_{r-1}\right\rangle \rightarrow \mathbb{C}[x, y] /\left\langle f_{x}, f_{y}\right\rangle
$$

induced by $g \mapsto g \cdot f$. Note that this map is well defined because $p_{i} f \in$ $\left\langle f_{x}, f_{y}\right\rangle$ for $i=1, \ldots, r-1$ (recall that $p_{i}$ 's are cofactors). The kernel of this map is $\left\langle f_{x}, f_{y}\right\rangle /\left\langle f_{x}, f_{y}, p_{1}, \cdots, p_{r-1}\right\rangle$ and the image is equal to $\left\langle f, f_{x}, f_{y}\right\rangle /\left\langle f_{x}, f_{y}\right\rangle$. Since the sum of the dimensions of the kernel and the image is equal to $\operatorname{dim} \mathbb{C}[x, y] /\left\langle f_{x}, f_{y}, p_{1}, \cdots, p_{r-1}\right\rangle$, the assertion follows.

Example: Let $r \geq 3$ and let $f=f_{1} \cdots f_{r}$ be homogeneous (thus $f$ has degree $r)$. Then,

$$
\operatorname{dim} \mathcal{V}_{f} / \mathcal{V}_{f}^{1} \geq \frac{(r-1)(r-2)}{2}
$$

To see this, note $f=x f_{x}+y f_{y} \in\left\langle f_{x}, f_{y}\right\rangle$; thus

$$
\operatorname{dim} \mathcal{V}_{f} / \mathcal{V}_{f}^{1}=\operatorname{dim}\left(\mathbb{C}[x, y] /\left\langle f_{x}, f_{y}, p_{1}, \cdots, p_{r-1}\right\rangle\right) .
$$

Since all generators of $\left\langle f_{x}, f_{y}, p_{1}, \cdots, p_{r-1}\right\rangle$ are homogeneous polynomials of degree $\geq r-2$, any set of polynomials of degree less than $r-2$ is linearly independent modulo $\left\langle f_{x}, f_{y}, p_{1}, \cdots, p_{r-1}\right\rangle$ if and only if it is linearly independent. (For $r=3$ one easily verifies that $\operatorname{dim} \mathcal{V}_{f} / \mathcal{V}_{f}^{1}=1$.)

\section{Computational issues}

In contrast with [2] and [10] we will discuss computational issues here, and we will illustrate that the computations are in fact feasible. For the problem of vector fields admitting the invariant curve $C$ we obtain precise information about the dimension modulo trivial vector fields, and an algorithm to determine a basis of $\mathcal{V}_{f} / \mathcal{V}_{f}^{0}$. All the necessary operations are standard procedures (e.g. for Gröbner bases), and many of these are built-in features of various computer algebra systems. The last step may be critical for some standard systems, but it uses only built-in features of a specialized system like Singular. [7] Let us describe the simple algorithmic nature of the approach in greater detail. The notation is as in Theorem 6 .

The algorithmic approach. Assume that a polynomial $f$ is given, with no multiple prime factors.

1. Let $f_{x}=s \cdot h_{1}, f_{y}=s \cdot h_{2}$ with a g.c.d. $s$ of the partial derivatives. (This is algorithmically accessible, e.g. via resultants.) Then (2) holds if and only if

$$
L=s \cdot \widehat{L} \quad \text { with } \quad \widehat{L} \in \mathbf{c}:\langle f\rangle .
$$

2. Let $G$ be a Gröbner basis of $\mathbf{c}$ with respect to some fixed monomial ordering. Then there are only finitely many monomials $m_{1}, \ldots, m_{d}$ which 
are not multiples of some leading monomial in $G$. According to Cox et al. [4], Ch. 5, §3, Prop. 1, the dimension of $\mathbb{C}[x, y] / \mathbf{c}$ is equal to the number of these monomials, and the classes $m_{i}+\mathbf{c}$ form a basis.

3. Now consider the map

$$
\begin{aligned}
M_{f}: \mathbb{C}[x, y] / \mathbf{c} & \rightarrow \mathbb{C}[x, y] / \mathbf{c} \\
g+\mathbf{c} & \mapsto f \cdot g+\mathbf{c}
\end{aligned}
$$

which, by definition, has the kernel $(\mathbf{c}:\langle f\rangle) / \mathbf{c}$. The matrix representation of $M_{f}$ with respect to the basis $\left(m_{i}+\mathbf{c}\right)$ can be obtained by fully reducing the polynomials $f \cdot m_{j}$ with respect to the Gröbner basis $G$, since this automatically yields a representation

$$
f \cdot m_{j} \equiv \sum_{i} \alpha_{i j} \cdot m_{i} \bmod (\mathbf{c})
$$

and thus the desired matrix $A=\left(\alpha_{i j}\right)$. Now standard linear algebra yields the kernel of $M_{f}$, and thus representatives of $(\mathbf{c}:\langle f\rangle) / \mathbf{c}$ as linear combinations of the $m_{i}$.

4. If some elements of $\mathbf{c}:\langle f\rangle$ are known, e.g. by way of (4), then one may replace $\mathbf{c}$ by some larger ideal $\mathbf{c}^{*}$, and determine a basis of $(\mathbf{c}:\langle f\rangle) / \mathbf{c}^{*}$.

5. From cofactors to vector fields: Every element of the Gröbner basis $G$ is a combination of $h_{1}$ and $h_{2}$. Given some $\widehat{L}=\sum \beta_{j} \cdot m_{j}$ from the kernel in $\underline{3}$. or $\underline{4}$. above, express $f \cdot \widehat{L}$ as a combination of the elements of $G$, and thus as a combination of $h_{1}$ and $h_{2}$. (Actually, the algorithm may run in a slightly different fashion; see Decker and Lossen [6], sections 2.2 and 3.6.) This yields the components of a vector field with cofactor $L=s \cdot \widehat{L}$.

In particular the algorithm allows us to easily decide whether some subspace is already equal to $\mathcal{V}_{f}$. We look at two examples (with degenerate singular points), in order to illustrate that the computations are indeed unproblematic. The computations were carried out using MAPLE and SiNGULAR.

Example 1. For the polynomial

$$
f=y \cdot\left(y-x^{2}\right) \cdot\left(x-y^{2}\right)
$$

we compute $\operatorname{dim} \mathcal{V}_{f} / \mathcal{V}_{f}^{0}$. One obtains the following Gröbner basis with respect to the grevlex order (using the terminology of [4]):

$$
\left\langle f, f_{x}, f_{y}\right\rangle=\left\langle y^{3}-2 x y+\underline{x^{3}},-x y^{2}+\underline{y^{4}}, y^{2}-3 x^{2} y+2 \underline{x y^{3}},-y^{3}+\underline{x^{2} y^{2}}\right\rangle .
$$

The leading monomials have been underlined. Now the monomials which are not multiples of some leading monomial above can readily be listed:

$$
1, x, x^{2}, y, x y, x y^{2}, y^{2}, x y^{2}, y^{3} \text {. }
$$


According to Corollary 7 the dimension of $\mathcal{V}_{f} / \mathcal{V}_{f}^{0}$ is equal to 9.

Example 2. Let $f=\left(y-x^{2}\right)\left(y-x^{3}\right)$. Then, with respect to the grevlex order, a Gröbner basis of $\left\langle f_{x}, f_{y}\right\rangle$ is given by

$$
-9 y^{2}+29 x y-40 y+20 \underline{x^{2}} ; 108 \underline{y^{3}}+85 x y-193 y^{2} ; 12 \underline{x y^{2}}+5 x y-17 y^{2}
$$

with leading monomials underlined. Therefore a basis of the quotient space is formed by the monomials

$$
1 ; x ; y ; x y ; y^{2}
$$

and reduction to normal form yields

$$
\begin{aligned}
f \cdot 1 & \equiv-\frac{1}{20} x y+\frac{1}{20} y^{2}, \\
f \cdot x & \equiv-\frac{1}{30} x y+\frac{1}{30} y^{2}, \\
f \cdot y & \equiv-\frac{1}{54} x y+\frac{1}{54} y^{2}, \\
f \cdot x y & \equiv-\frac{1}{81} x y+\frac{1}{81} y^{2}, \\
f \cdot y^{2} & \equiv-\frac{5}{729} x y+\frac{5}{729} y^{2} .
\end{aligned}
$$

Thus we obtain the matrix of $M_{f}$ :

$$
A=\left(\begin{array}{ccccc}
0 & 0 & 0 & 0 & 0 \\
0 & 0 & 0 & 0 & 0 \\
0 & 0 & 0 & 0 & 0 \\
-1 / 20 & -1 / 30 & -1 / 54 & -1 / 81 & -5 / 729 \\
1 / 20 & 1 / 30 & 1 / 54 & 1 / 81 & 5 / 729
\end{array}\right)
$$

and solving for the kernel shows that the polynomials

$$
2-3 x ; 5 x-9 y ; 2 y-3 x y ; 5 x y-9 y^{2}
$$

span the ideal of cofactors modulo $\left\langle f_{x}, f_{y}\right\rangle$.

We invoke additional knowledge according to $\underline{4 .:}$ The vector field $Y:=$ $f_{2} \cdot X_{f_{1}}$ admits $f$ with cofactor $L^{*}:=3 x^{2}-2 x$, and multiples of $Y$ will yield the corresponding multiples of $L^{*}$. Reduction to normal form yields

$$
\begin{aligned}
L^{*} \cdot 1 & \equiv-2 x+6 y-\frac{87}{20} x y+\frac{27}{20} y^{2}, \\
L^{*} \cdot x & \equiv-4 y+\frac{29}{4} x y-\frac{9}{4} y^{2}, \\
L^{*} \cdot y & \equiv-\frac{5}{4} x y+\frac{9}{4} y^{2},
\end{aligned}
$$


and $L^{*} \cdot x y \equiv L^{*} \cdot y^{2} \equiv L^{*} \cdot y$. Thus $\mathcal{V}_{f}^{1}$ has codimension one in $\mathcal{V}_{f}$, and only one additional vector field $Z=A \partial / \partial x+B \partial / \partial y$ with cofactor $2-3 x$ (for instance), needs to be found. Using Singular and proceeding with the lift command, exactly as in Decker and Lossen [6], subsection 3.6.1, one obtains

$$
\begin{aligned}
& A=-9 / 40 x^{5}+261 / 800 x^{4} y-1218891241 / 57600000 x^{3} y^{2}-27 / 40 x^{4} \\
& -580752454969 / 4976640000 x^{3} y-1218891241 / 57600000 x^{2} y^{2} \\
& -180423092156761 / 429981696000 x^{3}-580136595769 / 4976640000 x^{2} y \\
& -261 / 400 x y^{2}+1218891241 / 28800000 y^{3} \\
& -180444591241561 / 429981696000 x^{2}+9 / 10 x y \\
& +582376083769 / 2488320000 y^{2}+1 / 2 x+180229600393561 / 214990848000 y
\end{aligned}
$$

and

$$
\begin{aligned}
& B=-9 / 8 x^{6}+261 / 160 x^{5} y-1218891241 / 11520000 x^{4} y^{2}-9 / 4 x^{5} \\
& -581704237369 / 995328000 x^{4} y-783 / 800 x^{3} y^{2} \\
& +1218891241 / 19200000 x^{2} y^{3}-180229600393561 / 85996339200 x^{4}+9 / 5 x^{3} y \\
& +581293664569 / 1658880000 x^{2} y^{2}+1218891241 / 28800000 x y^{3}+1 / 2 x^{3} \\
& +180358594902361 / 143327232000 x^{2} y+582376083769 / 2488320000 x y^{2} \\
& +179907114121561 / 214990848000 x y+y .
\end{aligned}
$$

As this example illustrates, the algorithmic approach is feasible. As should be expected, the computer algebra system may produce quite large output. Modifying the vector field $X=A \frac{\partial}{\partial x}+B \frac{\partial}{\partial y}$ by elements of $\mathcal{V}_{f}^{1}$ of the form

$$
a\left(y-x^{2}\right)\left(\begin{array}{l}
1 \\
3 x^{2}
\end{array}\right)+b\left(y-x^{3}\right)\left(\begin{array}{l}
1 \\
2 x
\end{array}\right)
$$

with suitable $a$ and $b$ will yield $\tilde{X}=\tilde{A} \frac{\partial}{\partial x}+\tilde{B} \frac{\partial}{\partial y}$ where

$$
\begin{aligned}
& \tilde{A}=\frac{1}{4}\left(2 x-3 x^{4}+y\right), \\
& \tilde{B}=\frac{1}{4}\left(2 x^{3}+3 x^{5}+4 y-9 x^{3} y\right) ;
\end{aligned}
$$

which is more palatable.

Thus, while things are settled from an algorithmic prospective, more structural insight would be welcome.

\section{Sigma Processes}

In order to obtain a better structural understanding of the situation, blow ups may be helpful. For this purpose we turn to sigma processes; see Shafarevich [9]. Using a suitable finite succession of such processes one will break up singular points of multiplicity $>1$, as well as self-intersections of irreducible curves; thus one can reduce the geometry to the nondegenerate setting from Theorem 8. This follows from the Bendixson-Seidenberg 
theorem; see Anosov et al. [1]. The prototype (up to affine coordinate transformations) is described next.

Proposition 10. Consider the map

$$
\Phi: \mathbb{C}^{2} \rightarrow \mathbb{C}^{2}, \quad\left(\begin{array}{c}
x \\
y
\end{array}\right) \mapsto\left(\begin{array}{c}
x \\
x y
\end{array}\right) .
$$

(a) Given irreducible, pairwise relatively prime polynomials $f_{1}, \ldots, f_{r}$, with $f:=f_{1} \cdots f_{r}$, such that 0 is a singular point for $C$ and a polynomial vector field $X=(P, Q)$ on $\mathbb{C}^{2}$, the following are equivalent:

(i) The zero set of $f$ is invariant for $X$ with cofactor $K$.

(ii) The vector field

$$
\widehat{X}=\frac{1}{x} \cdot\left(\begin{array}{c}
x P(x, x y) \\
-y P(x, x y)+Q(x, x y)
\end{array}\right)
$$

is polynomial, and the zero set of $\hat{f}:=f(x, x y)$ is invariant for $\widehat{X}$ with cofactor $\widehat{K}(x, y)=K(x, x y)$.

(b) If $x$ is not among the prime factors of $f$ then $\hat{f}=f_{0}^{* s} \cdot f_{1}^{*} \cdots f_{r}^{*}$ with $f_{0}^{*}=x, f_{i}^{*}(x, y)=x^{-s_{i}} f_{i}(x, x y)$ irreducible for $1 \leq i \leq r$, and $s=s_{1}+\cdots+s_{r}>1$.

(c) For any polynomial $g$ one has

$$
\hat{X}_{g}=\frac{1}{x} X_{\hat{g}}
$$

(possibly with rational vector fields).

(d) If $X \in \mathcal{V}_{f}^{1}$ then $\hat{X} \in \mathcal{V}_{f^{*}}^{1}$, with $f^{*}:=f_{0}^{*} \cdot f_{1}^{*} \cdots f_{r}^{*}$.

Proof. Let $\hat{X}=\hat{P} \frac{\partial}{\partial x}+\hat{Q} \frac{\partial}{\partial y}$. Then statement (a) follows from the construction

$$
\left(\begin{array}{c}
\hat{P}(x, y) \\
\hat{Q}(x, y)
\end{array}\right)=D \Phi(x, y)^{-1}\left(\begin{array}{c}
P(\Phi(x, y)) \\
Q(\Phi(x, y))
\end{array}\right)
$$

which implies

$$
X(g) \circ \Phi=\hat{X}(g \circ \Phi)
$$

for all $g . \hat{X}$ is polynomial because 0 is a singular point of $f$, and therefore stationary for $X$.

For statement (b) note that $\hat{f}_{i}(x, y)=f_{i}(x, x y)=x^{s_{i}} f_{i}^{*}(x, y)$, with $x^{s_{i}}$ the highest power of $x$ that divides $\hat{f}_{i}$, and $f_{i}^{*}$ is irreducible. One has $s_{i}>0$ if and only if $f_{i}(0)=0$, and $s_{i}>1$ if and only if 0 is a singular point for $f_{i}$.

Statements (c) and (d) follow by elementary computations. (Note that, while $\frac{1}{x} X_{f_{i}^{*}}$ is rational whenever $f_{i}(0) \neq 0$, every $\frac{1}{x} \frac{f^{*}}{f_{i}^{*}} X_{f_{i}^{*}}$ is polynomial due to $s \geq 2$ ). 
We record the identities

$$
\begin{aligned}
& P(x, y)=\hat{P}\left(x, \frac{y}{x}\right), \\
& Q(x, y)=\frac{y}{x} \hat{P}\left(x, \frac{y}{x}\right)+x \hat{Q}\left(x, \frac{y}{x}\right),
\end{aligned}
$$

for later use. In principle one can use sigma processes to transfer complicated geometries in the affine plane to simpler ones, until Theorem 8(c) is applicable. The problem is to revert the blow-ups to the original setting, because there exist many vector fields that are not blow ups (as implied by (8)). Thus one gains general structural insight, but some problems remain.

To illustrate the applicability of blow-ups, we consider one (relatively simple) class of examples.

Proposition 11. Let $f=f_{1}$ be irreducible such that 0 is the only singular point of $C$, and assume that $f^{*}=x^{s} f_{1}^{*}$ satisfies the hypothesis of Theorem $8(c)$.

(a) Then every vector field $X$ that admits $f$ satisfies

$$
\hat{X}=A \cdot f_{1}^{*} \cdot\left(\begin{array}{c}
0 \\
1
\end{array}\right)+B \cdot x \cdot\left(\begin{array}{c}
-f_{1 y}^{*} \\
f_{1 x}^{*}
\end{array}\right)+x \cdot f_{1}^{*}\left(\begin{array}{c}
U \\
0
\end{array}\right)
$$

with suitable polynomials $A, B$ and $U$.

(b) One has

$$
\begin{aligned}
& P(x, x y)=x \cdot\left(-B f_{1 y}^{*}+U f_{1}^{*}\right) \\
& Q(x, x y)=x y \cdot\left(-B f_{1 y}^{*}+U f_{1}^{*}\right)+x \cdot\left(A f_{1}^{*}+B x f_{1 x}^{*}\right)
\end{aligned}
$$

and the cofactor $K$ of $f$ for the vector field $X$ satisfies

$$
K(x, x y)=s\left(-B f_{1 y}^{*}+U f_{1}^{*}\right)+A f_{1 y}^{*}+x U f_{1 x}^{*} .
$$

Proof. Statement (a) follows directly from Theorem 8(c); just note that any term $x \cdot f_{1}^{*} \cdot\left(\begin{array}{l}0 \\ V\end{array}\right)$ can be incorporated into the first one.

Statement (b) follows by straightforward computation.

One can put these results to practical use as follows. Setting $x=0$, one finds

$$
\begin{aligned}
K(0,0) & =s\left(-B(0, y) f_{1 y}^{*}(0, y)+U(0, y) f_{1}^{*}(0, y)\right)+A(0, y) f_{1 y}^{*} \\
& =(-s B(0, y)+A(0, y)) f_{1 y}^{*}(0, y)+s U(0, y) f_{1}^{*}(0, y) .
\end{aligned}
$$

So the right hand side of this identity must be constant. Generally, there is a systematic test whether a polynomial $h(x, y)$ can be written as $g(x, x y)$, with some polynomial $g$ : Setting $h=\sum_{\ell \geq 0} h_{\ell}(y) x^{\ell}$, the necessary and sufficient condition is that $h_{l}$ has degree $\leq \ell$ for all $\ell$.

Thus one may proceed to evaluate the identities in Proposition 11(b) degree by degree in $x$. This is a heuristic approach, not an algorithm (since 
termination is not guaranteed). But the heuristic turns to be useful in several instances.

Example 1. Consider $f(x, y)=x^{2}+x^{m} y^{n}-y^{2}$, with $m \geq 2$ and $m+n \geq$ 3 . This polynomial is irreducible, and we have only one singular point at $(0,0)$, with $\operatorname{dim} \mathcal{V}_{f} / \mathcal{V}_{f}^{0}=1$. Note the remarks preceding Theorem 8. Now $\hat{f}(x, y)=x^{2}\left(1+x^{m+n-2} y^{n}-y^{2}\right)$, and if $X$ admits $f$ then

$$
\begin{aligned}
\hat{X} & =A \cdot\left(1+x^{m+n-2} y^{n}-y^{2}\right) \cdot\left(\begin{array}{c}
0 \\
1
\end{array}\right) \\
& +B \cdot x \cdot\left(\begin{array}{c}
2 y-n x^{m+n-2} y^{n-1} \\
(m+n-2) x^{m+n-3} y^{n}
\end{array}\right)+x\left(1+x^{m+n-2} y^{n}-y^{2}\right) \cdot\left(\begin{array}{c}
U \\
0
\end{array}\right) .
\end{aligned}
$$

Assuming $A=0$ we get

$$
\begin{aligned}
K(x, x y)= & 2 B \cdot\left(2 y-n x^{m+n-2} y^{n-1}\right)+2 U \cdot\left(1+x^{m+n-2} y^{n}-y^{2}\right) \\
& +x \cdot U \cdot(m+n-2) x^{m+n-3} y^{n},
\end{aligned}
$$

thus

$$
K(0,0)=2 B(0, y) \cdot 2 y+2 U(0, y) \cdot\left(1-y^{2}\right) .
$$

The simplest choice is $B(0, y)=y, U(0, y)=2$. Trying $B=y$ and $U=2$ (i.e., $B$ and $U$ are independent of $x$ ) we find

$$
K(x, x y)=4+(6-2 n) x^{m+n-2} y^{n}
$$

so

$$
K(x, y)=4+(6-2 n) x^{m-2} y^{n}
$$

and moreover

$$
X=\left(\begin{array}{l}
2 x+(2-n) x^{m-1} y^{n} \\
2 y+m x^{m-2} y^{n+1}
\end{array}\right)
$$

by elementary computation. (Note that there must be a cofactor $K$ with $K(0,0) \neq 0)$, according to Theorem 8 . There is no general guarantee that the heuristic works with $B$ and $U$ functions of $y$ alone; but this would be the first attempt in any sense. If necessary, incorporate higher $x$-powers.)

Example 2. We consider $f(x, y)=y(x+y)(x-y)+x^{4}$. This polynomial is irreducible, and by computations as in Section 4 Example 2, one finds that $\operatorname{dim} \mathcal{V}_{f} / \mathcal{V}_{f}^{0}=4$, and that the polynomials

$$
1+6 y ; \quad x(1+6 y) ; \quad y(1+6 y) ; \quad y^{2}(1+6 y)
$$

span the ideal of cofactors modulo $\left\langle f_{x}, f_{y}\right\rangle$. Rather than taking the algorithmic approach, we use sigma processes here in order to find vector fields. 
We have $\hat{f}=x^{3}\left(y-y^{3}+x\right)=x^{3} f_{1}^{*}$, thus $f^{*}$ satisfies Theorem 8(c) and a vector field admitting $f^{*}$ has the form

$$
A \cdot\left(y-y^{3}+x\right)\left(\begin{array}{l}
0 \\
1
\end{array}\right)+B x \cdot\left(\begin{array}{c}
3 y^{2}-1 \\
1
\end{array}\right)+x\left(y-y^{3}+x\right) \cdot\left(\begin{array}{c}
U \\
0
\end{array}\right) .
$$

Assuming $A=0$, we obtain

$$
K(x, x y)=3\left(B(0, y) \cdot\left(3 y^{2}-1\right)+U \cdot\left(y-y^{3}+x\right)\right)+x U .
$$

Therefore $K(0,0)=3\left(B(0, y) \cdot\left(3 y^{2}-1\right)+U(0, y) \cdot\left(y-y^{3}\right)\right)$. The Euclidean algorithm suggests

$$
B(0, y)=\frac{3}{2} y^{2}-1, \quad U(0, y)=\frac{9}{2} y .
$$

With the working hypothesis $B_{x}=U_{x}=0$, we have $B(x, y)=\frac{3}{2} y^{2}-1$, $U(x, y)=\frac{9}{2} y$, and $K(x, x y)=3\left(1+\frac{9}{2} y\right)=3+18 x y$, which yields

$$
K(x, y)=3(1+6 y) .
$$

By elementary computations the vector field

$$
\tilde{Y}=\left(\frac{3}{2} y^{2}-1\right) x \cdot\left(\begin{array}{c}
3 y^{2}-1 \\
1
\end{array}\right)+x\left(y-y^{3}+x\right) \cdot\left(\begin{array}{c}
\frac{9}{2} y \\
0
\end{array}\right)
$$

turns out to be the blow-up of

$$
Y=\left(\begin{array}{l}
x+\frac{9}{2} x y \\
y+6 y^{2}-x^{2}
\end{array}\right) .
$$

Thus we have found $Y(f)=3(1+6 y) \cdot f$; and this solves our problem: The vector field $Y$ provides the cofactor $1+6 y$ (up to a scalar); Thus $x \cdot Y, y \cdot Y$ and $y^{2} \cdot Y$ will give the remaining cofactors.

These examples are not sufficient to decide about the relative merit of sigma process versus the algorithmic approach. But they indicate that further work with sigma processes maybe promising.

\section{REFERENCES}

[1] D.V. Anosov et al.: Ordinary differential equations and smooth dynamical systems. Springer-Verlag, Berlin 1997.

[2] C. Christopher, J. Llibre, Ch. Pantazi, S. Walcher: Inverse problems for multiple invariant curves. Proc. Royal Soc. Edinburgh (to appear).

[3] C. Christopher, J. Llibre, Ch. Pantazi, X. Zhang: Darboux integrability and invariant algebraic curves for planar polynomial systems. J. Phys. A 35 (2002), 2457-2476.

[4] D. Cox, J. Little, D. O'Shea: Ideals, varieties and algorithms, 2nd ed. SpringerVerlag, New York 1997.

[5] D. Cox, J. Little, D. O'Shea: Using algebraic geometry. Springer-Verlag, New York 1998.

[6] W. Decker, C. Lossen: Computing in algebraic geometry. A quick start using SINGULAR. Springer-Verlag, Berlin 2006. 
[7] G. M. Grevel, G. Pfister, H. Schönemann: Singular 3.0. A computer algebra system for polynomial computations. Centre for Computer Algebra, University of Kaiserslankern (2005) http://www.singular.uni-kl.de

[8] Ch. Pantazi: Inverse problems of the Darboux theory of integrability for planar polynomial differential systems. Doctoral thesis, Universitat Autonoma de Barcelona 2004.

[9] I.R. Shafarevich: Basic algebraic geometry. Springer-Verlag, Berlin 1977.

[10] S. Walcher: Plane polynomial vector fields with prescribed invariant curves. Proc. Royal Soc. Edinburgh 130A (2000), 633-649.

[11] S. Walcher: On the Poincaré problem. J. Differential Eqs. 166 (2000), 51-78.

1 Department of Mathematics and Statistics, University of Plymouth, PlyMOUTH PL2 3AJ, U.K.

E-mail address: c.christopher@plymouth.ac.uk

2 Departament de Matemàtiques, Universitat Autònoma de Barcelona, 08193 Bellaterra, Barcelona, Spain

E-mail address: jllibre@mat.uab.cat

3 Departament de Matemàtica Aplicada I, Universitat Politècnica de Catalunya, ETSEiB, Av. Diagonal, 647, 08028 Barcelona, Spain

E-mail address: Chara.Pantazi@upc.edu

${ }^{4}$ Lehrstuhl A für Mathematik, RWTH Aachen, 52056 Aachen, Germany

E-mail address: walcher@mathA.rwth.aachen.de 